\title{
A Hybrid Method Based on Semi-Supervised Learning for Relation Extraction in Chinese EMRs
}

Chunming Yang ( $\nabla$ yangchunming@swust.edu.cn )

Southwest University of Science and Technology

Dan Xiao

Southwest University of Science and Technology

Yuanyuan Luo

Southwest University of Science and Technology

Bo Li

Southwest University of Science and Technology

\section{Xujian Zhao}

Southwest University of Science and Technology

Hui Zhang

Southwest University of Science and Technology

\section{Research Article}

Keywords: semi-supervised learning, relation extraction, medical knowledge graphs, residual network

Posted Date: February 24th, 2022

DOI: https://doi.org/10.21203/rs.3.rs-1357125/v1

License: (c) (i) This work is licensed under a Creative Commons Attribution 4.0 International License.

Read Full License 


\title{
A Hybrid Method Based on Semi-Supervised Learning for Relation Extraction in Chinese EMRs
}

\author{
Chunming Yang ${ }^{1,3^{*}}$, Dan Xiao ${ }^{1}$, Yuanyuan $\mathrm{Luo}^{1}, \mathrm{Bo} \mathrm{Li}^{1}, \mathrm{Xujian} \mathrm{Zhao}^{1}$ and Hui Zhang ${ }^{2}$ \\ ${ }^{1}$ School of Computer Science and Technology, Southwest University of Science and Technology,Mianyang \\ 621010, Sichuan, China \\ ${ }^{2}$ Southwest University of Science and Technology,School of Science, Mianyang 621010, Sichuan, China \\ ${ }^{3}$ Sichuan Big Data and Intelligent Systems Engineering Technology Research Center, Mianyang 621010, \\ Sichuan, China
}

\begin{abstract}
Background: Building a large-scale medical knowledge graphs needs to automatically extract the relations between entities from electronic medical records(EMRs). The main challenges are the scarcity of available labeled corpus and the identification of complexity semantic relations in text of Chinese EMRs. A hybrid method based on semi-supervised learning is proposed to extract the medical entity relations from small-scale complex Chinese EMRs.

Methods: The semantic features of sentences are extracted by a residual network(ResNet) and the long dependent information is captured by bidirectional GRU(Gated Recurrent Unit). Then the attention mechanism is used to assign weights for the extracted features respectively, and the output of two attention mechanisms is integrated for relation prediction. We adjusted the training process with manually annotated small-scale relational corpus and bootstrapping semi-supervised learning algorithm, and continuously expanded the datasets during the training process.

Results: We constructed a small corpus of Chinese EMRs relation extraction based on the EMR datasets released at the CCKS(China Conference on Knowledge Graph and Semantic Computing). The experimental results show that the best F1-score of the proposed method on the overall relation categories reaches $89.78 \%$, which is $13.07 \%$ higher than the baseline CNN.
\end{abstract}

Keywords: semi-supervised learning; relation extraction; medical knowledge graphs; residual network

\section{Background}

EMRs are digital information generated by medical staff using electronic systems, such as text, symbols, charts, data, and images [1]. Among them, unstructured texts (such as discharge summaries, medical records, surgical records, pathology reports, etc.) are a major part of EMRs, which are conducive to accurately describing the medical process. By identifying various named entities and the relations between them that are closely related to patients in EMRs, we can obtain valuable medical knowledge and patient health information [2]. For example, in ”患者3月前因直肠癌 于在我院于全麻上行直肠癌根治术, 手术过程顺利, 术 后给予抗感染及营养支持治疗, 患者恢复好。[The patient suffered from rectal cancer 3 months ago in our hospital under general anesthesia for radical resection of rectal cancer (DIXON), the operation process went

${ }^{*}$ Correspondence: yangchunming@swust.edu.cn

${ }^{1}$ School of Computer Science and Technology, Southwest University of Science and Technology, Mianyang 621010, Sichuan, China

Full list of author information is available at the end of the article smoothly, the postoperative anti-infection and nutritional support treatment was given, and the patient recovered well.]", "直肠癌 [rectal cancer]" is a disease name, "直肠癌根治术 [DIXON]" is a treatment method (the two are called named entities in the relation extraction research), and the relation between them is "改善", which belongs to the "TrAD" relationship in Table 2. We obtain a piece of medical knowledge that "直肠癌根治术改善了直肠癌 [DIXON improved rectal cancer]". We can get a lot of such knowledge from EMRs and build a professional medical knowledge base, which is of great significance for promoting the establishment of a clinically-assisted decision system, personal health model, and intelligent medical QA(Question and Answer) [3].

Previous deep learning technologies have made important contributions in the field of relation extraction, but most studies only use a single CNN or RNN network as a feature extractor, and few studies use deep networks to complete relation extraction in Chi- 
nese EMRs. Different from the news corpus commonly used in the open-domain field, Chinese EMRs have unique linguistic features, including a large number of long sentences and medical professional vocabularies. And the shallow neural network cannot well extract the complex semantic features in the text of EMRs. On the other hand, there is a lack of Chinese EMRs relation labeled corpus,and other language resources, so most of the supervised and distant supervised methods are not suitable. Based on the above problems, we proposed a hybrid relation extraction method based on semi-supervised learning. This method combines the advantages of the deep residual network and GRU so that the model can fully learn the features of different levels and long-term dependency. Then we use the attention mechanism to further strengthen the key information. Secondly, and used the bootstrapping semisupervised learning algorithm to adjust the training process. Experimental results show that our method can accurately extract relations in Chinese electronic medical records with only a small amount of labeled data, with the overall F1-score reaching $89.78 \%$.

\section{Literature Review}

Relation extraction is essentially a classification task, i.e., classifying target entity pairs and sentences containing entity pairs according to the pre-defined relation categories. The previous relation extraction studies in open-domain filed mainly adopted feature engineering or kernel function method, which had poor classification performance, and required a lot of manpower to construct the feature set $[4,5,6]$.

As the usage scenarios of deep learning become more and more extensive, many researchers apply neural network to relationship extraction tasks. The commonly used models include CNN [7, 8], RNN [9] and its variant LSTM [10]. RNN can effectively learn the context dependence of text sequences, but it can not capture the features at the syntactic and semantic levels. CNN can capture the local information in the sentence, but ignores the role of global information. Zeng et al.[11] exploited Piecewise Convolutional Neural Networks (PCNNs) on the task of relation extraction and incorporated multi-instance learning to address the mislabeling problem. Lin et al.[12] proposed a convolutional neural network (CNN) architecture with sentence-level selective attention for distant supervised relation extraction, which can make full use of all informative sentences and reduce the weights of those noisy instances. Considering the different contribution of every single pair of relational semantics in the sentence, researchers have introduced the attention mechanism, combined it with CNN and LSTM respectively, and achieved good results. Zhou et al.[13] combined
Bidirectional Long Short-Term Memory (LSTM) and multiple attention mechanisms for relation classification. Experimental results on the SemEval-2010 Task8 datasets show that this method outperforms most methods with only word vectors.

Deep residual learning (ResNet) [14] is a new method for training very deep neural networks using identity mapping for shortcut connections. However, the effect of residual learning on noisy natural language processing tasks is still not well understood. Zhang et al.[2] Proposed an Attention-Based Deep Residual Network (ResNet) to recognize medical concept relations in Chinese EMRs. The model achieved a F1score of $77.80 \%$ on the manually annotated Chinese EMRs corpus and outperforms the state-of-the-art approaches. It shows that The residual network-based model can reduce the negative impact of corpus noise on parameter learning, and the combination of character position attention mechanisms will enhance the identification features of different entities.

GRU (Gated Recurrent Unit) is a commonly used gated RNN. Due to its relatively simple structure, GRU has a faster computing speed than LSTM; Moreover, due to fewer parameters, GRU has a better generalization effect on small sample data; The combination of GRU and other methods has also achieved good results in different fields. Hong et al.[15] adopted the method of relationship extraction based on Bidirection Gated Recurrent Unit (BiGRU) and attention mechanism (BiGRU-ATT) to retrieve these relations from Chinese medical text. The experimental results show that regarding Chinese medical entity relationship extraction, they can achieve a better accuracy and recall than using a convolutional neural network(CNN).

In 2010, the i2b2/VA NLP challenge for clinical Records proposed the medical entity relation extraction task with English EMRs, focused on assigning three relation categories that hold between medical problem, test, and treatment [16]. In recent years, the CCKS conference has released Chinese EMRs named entity sharing tasks and annotated datasets since 2017, which has greatly promoted the research of Chinese medical information extraction. Fenia et al.[17] proposed an end-to-end method for the relation classification between drugs and drug-related entities. This method integrated neural network models such as bidirectional LSTM, attention mechanism and transform, which could simultaneously extract the relations within and between sentences. Xu et al.[18] utilized a data-driven framework to extract structured records from the free-text narrative, with an F1-score of $84.6 \%$ on 24,817 Chinese EMRs datasets. Zhang et al.[19] proposed a semi-supervised biomedical relation extraction method based on Variational Autoencoder (VAE), 
and the datasets of PPI, DDI, CPI were used to evaluate this method. Song et al.[20] used the graphical neural network (GNN) to generate high-quality dependent forests and solved the problem of low accuracy of dependent analysis in the biomedical relation extraction by taking dependent forests as external features. Liu et al.[21] proposed a capsule network model combining the shortest dependent path, and the F1-score of this model on the DDI Extraction 2013 datasets was $1.17 \%$ higher than that of the current best model.

CNN and RNN have always been the baseline models of relationship extraction, and researchers have constantly innovated CNN and RNN. ResNet and BiGRU are widely used in relationship extraction tasks. However, in the field of Chinese medicine, the feature extraction ability of them are still slightly insufficient to capture the complex semantic information in EMRs text. To solve the above problems, we propose a hybrid neural network relationship extraction model based on residual network, gated loop unit and attention mechanism. Experiments show that our model achieves the best effect on our manually annotated Chinese EMRs corpus.

We make the following major contributions in this work:

- We propose a hybrid neural network model based on semi-supervised learning to extract relationships in Chinese EMRs.

- Our proposed hybrid neural network model achieves better results in performing semantic extraction, which cannot be achieved by other current models.

- The semi-supervised learning approach we take proves to be effective in expanding the data on a small annotated corpus.

\section{Methods}

Relation Extraction Model

We propose the ResGRU-Att for relation extraction in Chinese EMRs. The model is composed of an embedding layer, a hybrid neural network layer (including residual network, BiGRU, attention mechanism), and a classification layer. The overall architecture of our relation extraction model is shown in Figure 1.

\section{Embedding Layer}

For a given sentence $S=\left\{c_{1}, c_{2}, \ldots, c_{i}, \ldots\right\}$, including the marked entity pair $e=\left[e_{1}, e_{2}\right]$. Each character $c_{i}$ in the sentence is mapped to a character embedding and two-position embedding. After the two vectors are spliced, The final vector representation of each character $x_{i}=\left[x_{i w}, x_{i p}\right]$ is obtained by splicing these two embedding vectors. character embedding We use the word2vec tool to pre-train all medical record texts, and then use it to initialize the character embedding of the input sentence.

position embedding In the task of relation extraction, the words close to the target entities are usually informative to determine the relation between entities. Similar to Zeng et al.[22], position embedding reflects the positions of target entity pair and the relative distance between characters and the marked entity pair. Each character $c_{i}$ is mapped into two position embedding through random initialization. Figure 2 gives an example of the relative distance between a character and two entities, where the relative distances between “形” and "双膝关节” and "膝关节性关节炎" are 7 and -5 , respectively.

The input of model is the matrix $X$ :

$$
X=\left[x_{1}, x_{2}, \ldots, x_{i}, \ldots\right] \in R^{L \times d_{v}}
$$

Here, $x_{i}$ represents the vector representation of the $\mathrm{i}$-th character in sentence, and $L$ is length. We set the dimension of character embedding to $d_{w}$ and the dimension of position embedding to $d_{p}$, and the vector dimension of the sentence is $d_{v}=d_{w}+2 d_{p}$.

\section{Hybrid neural network layer}

The hybrid neural network layer combines the features extracted by ResNet and BiGRU. While increasing the network depth, it ensures the model's ability to learn local information and long-term dependency. Then the attention mechanism is used to further highlights significant information for relation classification.

\section{ResNet}

ResNet [23] consists of a standard convolutional neural network and four residual convolution blocks. Assuming that the vector matrix of consecutive $h$ characters from the i-th character in the sentence $\mathrm{S}$ is $x_{i: i+h-1}$, use the filter $W \in R^{h \times d_{v}}$ to perform convolution operation on $x_{i: i+h-1}$ to obtain the feature $c_{i}$ in the window $h$ as (2) shown:

$$
c_{i}=f\left(w \cdot x_{i: i+h-1}\right)
$$

Here, $w$ represents the weight parameter matrix of the filter, $b$ is the bias term, and $f$ is a nonlinear function.

ResNet uses a shortcut connection based on the standard convolutional network, which connects the output characteristics of the underlying network to the high-level. Shortcut connection strengthens the multiplexing and transmission of features between different 


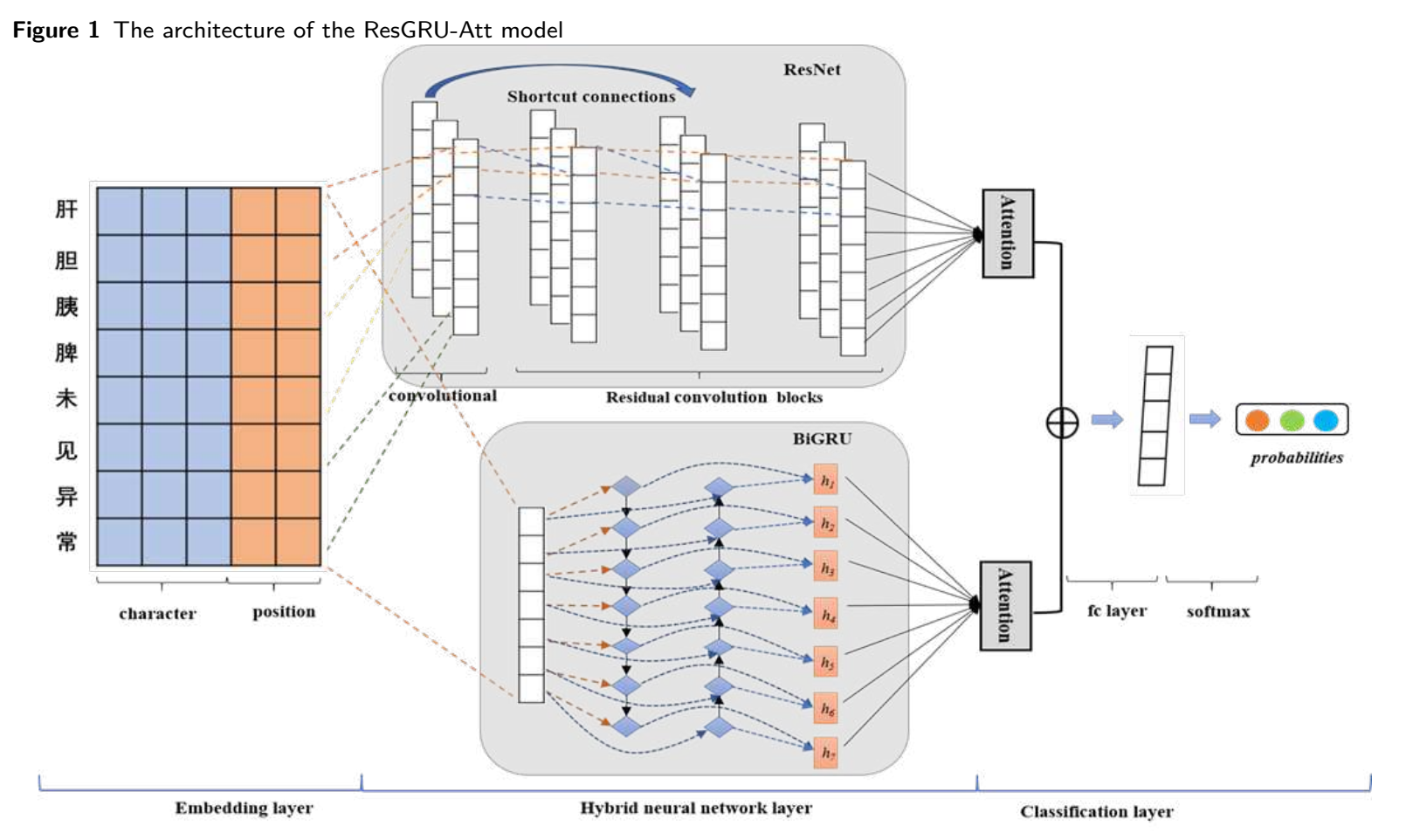

Figure 2 Example of position embedding

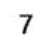

患者出现双膝关节外翻畸形，诊断为膌关节性关节炎。
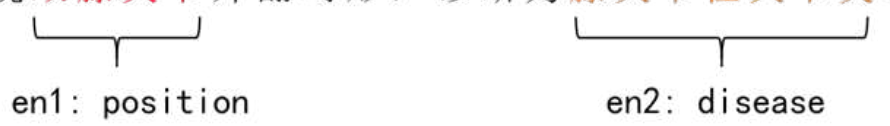

Figure 3 Residual convolution block

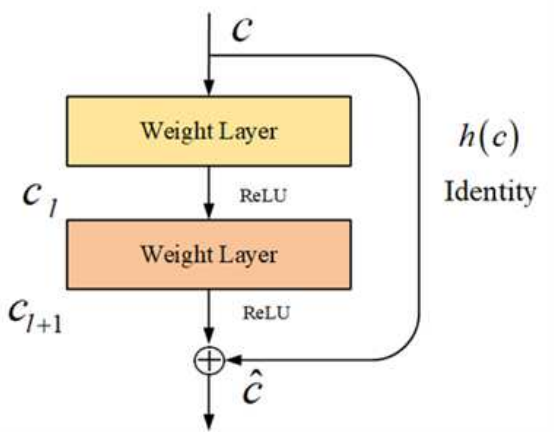

levels and avoids the problem of network degradation and gradient disappearance caused by too many layers. The structure of residual convolution block is shown in
Figure 3. Each block contains two convolutional layers. The ReLU function is used to activate the neuron after each convolutional layer. The features are directly passed to the next layer to realize shortcut connections between different residual convolution blocks by identity mapping.

Assuming that the input of residual convolution block is $c$, the output of block is expressed as:

$$
\begin{aligned}
& c_{l}=f\left(w_{1} \cdot c+b_{1}\right) \\
& c_{l+1}=f\left(w_{2} \cdot c_{l}+b_{2}\right) \\
& \hat{c}=g\left(c_{l}+h(c)\right)
\end{aligned}
$$

Here, $c_{l}$ and $c_{l+1}$ represent output of the first convolution and the second, $c$ is output of the residual convolution block. $w_{1}, w_{2} \in R^{h \times 1}$ are the weight parameter matrices of the two convolution filters. $b_{1}$ and $b_{2}$ are paranoid terms, $f$ and $g$ are activation functions. $h(c)=c$ is the identity mapping function, which 
is used to directly transfer the output features of the current layer to the next layer of the network.

\section{$B i G R U$}

GRU is a variant of RNN that uses a gate structure to learn long-term dependent information, which can effectively solve the problems of gradient disappearance and explosion in RNN. Compared with LSTM, GRU has fewer training parameters and speeds up. The GRU unit structure is shown in Figure 4.

\section{Figure 4 GRU unit}

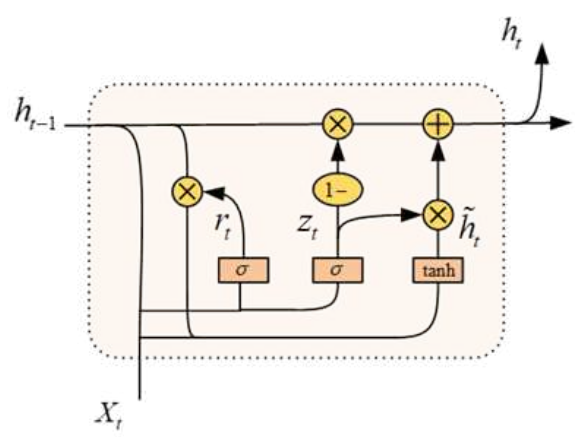

Suppose the current time is $t, X_{t}$ is the character processed by the GRU unit at time $t, h_{t-1}$ is the hidden state at the previous time, and $h_{t}$ is the hidden state at the current time. The update method of the GRU unit is:

$$
\begin{aligned}
& z_{t}=\sigma\left(W_{z}\left[h_{t-1}, X_{t}\right]+b_{z}\right) \\
& r_{t}=\sigma\left(W_{r}\left[h_{t-1}, X_{t}\right]+b_{r}\right) \\
& h_{t}=\tanh \left(W_{h}\left[h_{t-1}, X_{t}\right]+b_{h}\right) \\
& \left(1-z_{t}\right) \otimes h_{t-1}+z_{t} \otimes \widetilde{h_{t}}
\end{aligned}
$$

Here, $z_{t}$ and $r_{t}$ represent the update gate and the reset gate respectively. The update gate determines the information passed from the previous moment to the current, and the reset gate determines the information that was discarded in the hidden state at the previous moment. $h_{t}$ is the candidate's hidden state. $W_{z}, W_{r}$ and $W_{h}$ represent weight parameters at time $t$, and $b_{z}$, $b_{r}$, and $b_{h}$ are bias vectors. $\sigma$ and tanh are activation functions. $\otimes$ is a matrix cross-product operation.

We use a bidirectional GRU (BiGRU) structure to calculate an input sequence at the same time, and stitch the two hidden states to obtain the final out $h_{t}=\left[\overrightarrow{h_{t}}, \overleftarrow{h_{t}}\right]$

\section{Attention mechanism}

In the actual relationship extraction task, different characters are not equally important to judge the relationship type, and the decisive information may appear in any position of the sentence. The attention mechanism is introduced into the hybrid neural network layer to assign different weights to each character in the sentence, to emphasize the information that plays a key role in relationship extraction and reduce the interference of other irrelevant information. The calculation formula is as follows:

$$
\begin{aligned}
& a_{i}=\operatorname{Softmax}\left(\tanh w \cdot H_{i}+b\right) \\
& S=\sum a_{i} \cdot H_{i}
\end{aligned}
$$

Where $H_{i}$ represents the input. $a_{i}$ is the attention weight given to the i-th character in the sentence.

Firstly, the results of residual network and BiGRU are calculated respectively, then the two attention scores are fused to obtain the final output of the hybrid neural network layer:

$$
S=\left(S_{c} \oplus S_{G}\right)
$$

Where $S_{c}$ is the attention score of the residual network, $S_{G}$ is the attention score of BiGRU, and $S$ is the attention score of the hybrid neural network.

\section{Classification layer}

The final classification layer sends the features into a fully connected layer and a SoftMax classifier to complete the relation classification. The SoftMax classifier is an $r$-dimensional vector, where $r$ is the number of relation categories, and the value of vector represents the probability of a relation category.

\section{Semi-supervised learning}

To make use of the large amount of unlabeled data, a semi-supervised approach is the most appropriate. And we use the most widely used bootstrapping (see Table 1) method to learn. The basic idea is to use a small amount of seed datasets to select the highest score instance from unlabeled data, which significantly reduces the cost of manual labeling.

We use the bootstrapping to adjust the training process of relation extraction. The specific steps are:

Table 1 Bootstrapping algorithm

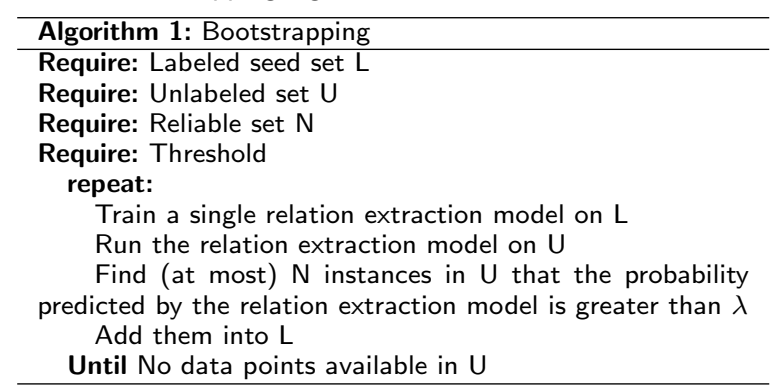




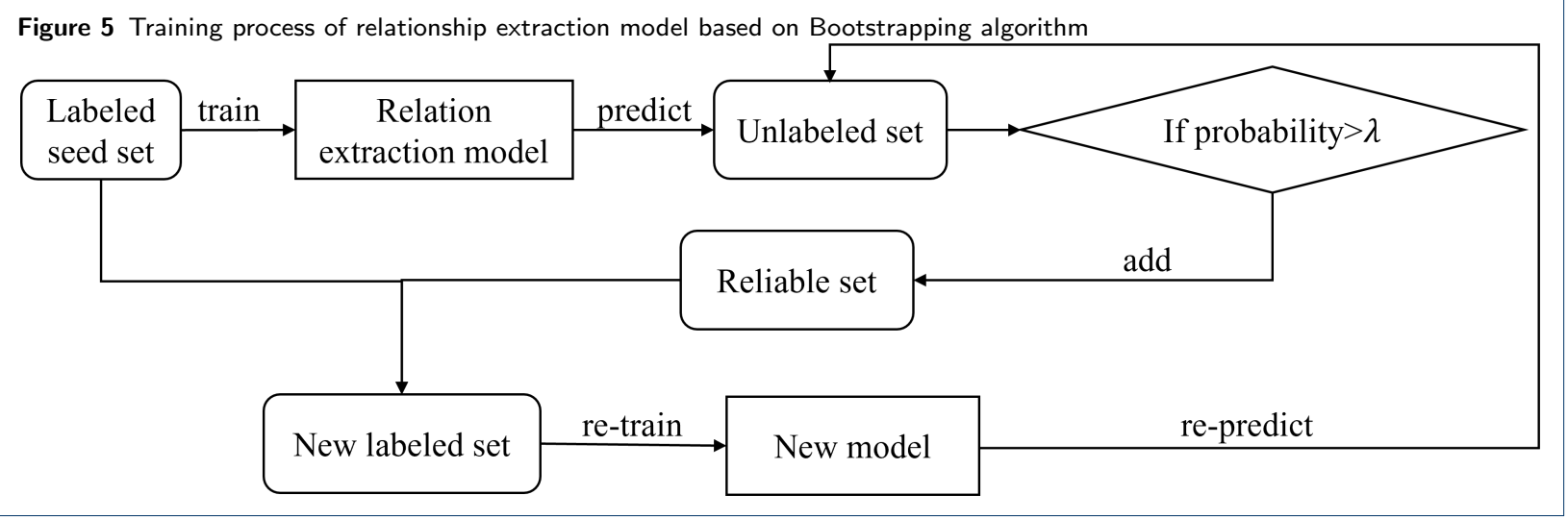

As shown in Figure 5, the bootstrapping is used to adjust the training process of relation extraction. The specific steps are as follows:

(1) First, use a small amount of manually labeled data as a seed set to train an initial relation extraction model (O-Relation).

(2) Use the O-Relation Model to predict the Unlabeled set, and output a relation label and a probability corresponding to the label for each piece of data. If the probability is greater than the threshold $(\lambda=0.7)$, this data is divided into the reliable set.

(3) When the number of reliable sets reaches 1000 , the seed set and the authentic set are merged into a new labeled datasets, and a new relation extraction model (B-relation) is re-trained.

(4) Repeat steps (2) and (3) until the unlabeled data set is cleared.

\section{Data Collection and Processing}

In this paper, we built a small-scale Chinese EMRs relation corpus by manual tagging from CCKS in 2017 [24], 2019 [25], and 2020[26]. We established a Chinese EMR relation annotation standard [1] are shown in Table 2, which includes 7 categories of entity pairs and 11 relation. The entities are divided into the five categories of treatment, disease, symptom, test, and position. Our corpus has marked all entities and a small number of relations, so subsequent experiments do not need to perform named entity recognition tasks. In the end, we constructed contains 75,000 sentences, 37,000 entities and 7,000 entity relations.

Figure 6 shows an example of relation extraction. First, all the entities in a sentence (separated by periods) are grouped into pairs of entities according to their possible relationships. We train a classifier to predict which category of relationships the entity pair belongs to. If there is no relationship, it will be marked as "unknown". We believe that "unknown" is a special relationship and will not be calculated in the final experiment. For example, there is indeed a relationship between "胃镜[gastroscopy]" (This is a test entity.) and "胃窦癌 [gastric antrum cancer]" (This is a disease entity.), and the relationship between these two entities is "TeRD"( Test reveals the disease.). However, "Xeloda (treatment)" and "gastric antrum cancer (disease)", for example, are not actually related to each other and will be marked as "Unknown".

At the stage of bootstrapping label classification, 1000 labeled data would be generated in each iteration, so we divided the training set and test set in the ratio of $8: 2$ in the iteration stage for subsequent experiments. And if there is no special explanation, the subsequent experiment is to calculate the overall F1 value of the whole relationship.

\section{Models, Parameters and Evaluation metrics}

We compared the ResGRU-Att with several neural network models and variants of the ResGRU described in the following.

CNN [22]: The CNN is baseline in whole experiment which contains a convolutional layer and a maxpooling layer.

CNN-Att: Based on the CNN, this model uses a character-level attention mechanism instead of the maximum pooling layer to aggregate the features.

PCNN [11]: The model divides one sentence into three pieces by the positions of two entities, and uses piece max-pooling to aggregate the features extracted from CNN.

ResNet [23]: The model consists of a convolutional network, four residual convolution blocks and a maximum pooling layer.

BiLSTM-Att/BiGRU-Att [13]: The two models use bidirectional LSTM and bidirectional GRU as feature extractors respectively, and then connect an attention mechanism. 
Table 2 Relation annotation standard

\begin{tabular}{|c|c|c|c|c|}
\hline Entity pair category & Number of entity pairs & Relation category & Number of relations & Relation description \\
\hline Disease-Position & 16538 & DAP & 304 & Disease is applied to the position in the body \\
\hline & & SAP & 518 & Symptom is applied to the position in body \\
\hline Symptom-Position & 19280 & SNAP & 893 & Symptom is not applied to the position in the body \\
\hline Test-Disease & 5743 & TeRD & 342 & Test reveals the disease \\
\hline Test-Position & 30673 & $\begin{array}{l}\text { TeAP } \\
\text { TeCP }\end{array}$ & $\begin{array}{c}1194 \\
572\end{array}$ & $\begin{array}{l}\text { Test is applied to the position in body } \\
\text { The results of the test contains the position in the body }\end{array}$ \\
\hline Test-Symptom & 13617 & $\begin{array}{l}\text { TeRS } \\
\text { TeAS }\end{array}$ & $\begin{array}{l}190 \\
110\end{array}$ & $\begin{array}{l}\text { Test reveals the symptom } \\
\text { Test is applied to the symptom }\end{array}$ \\
\hline Treatment-Disease & 5629 & $\begin{array}{l}\text { TrAD } \\
\text { TrRD }\end{array}$ & $\begin{array}{l}679 \\
227\end{array}$ & $\begin{array}{l}\text { Treatment is applied to the disease } \\
\text { Treatment (mainly surgery) reveals the disease }\end{array}$ \\
\hline Treatment-Position & 8871 & TrAP & 128 & Treatment is applied to the position in body \\
\hline
\end{tabular}

Figure 6 Example of relation extraction

, 缘于入院前 5 月余因“反复返酸、嗳气7年”就诊我 院, , 行胃镜检查示: “1. 胃㪡癌2. 慢性萎缩性胃 炎”, , 病理示: “胃管状腺癌II-III级”, 下腹部平扫 增强示: “胃肿瘤, 伴腹腔、腹膜后多发淋巴结䛲 大”, 考虑肿瘤局部较晚期, 遂于2015.04.18、 $2015.05 .11 、 2015.06 .02 、 2015.06 .29 、 2015.08 .02$ “安素泰210MGIVGTTD1+奥沙利铂（艾恒） 150MGIVGTTD2+希罗达1000MGBIDPOD1-14”方案 新辅助化疗、辅以止吐、制酸、保胃、保肝、营养 支持等治疗。

(, the reason is that more than 5 months before admission, he visited our hospital due to "repeated acid reflux and belching for 7 years". Gastroscopy showed: "1. Gastric antrum cancer 2. Chronic atrophic gastritis", pathology showed: "gastric tubular adenocarcinoma grade II-III", plain scan + of lower abdomen and enhancement showed: "gastric tumor with multiple lymphadenopathy of abdominal cavity and retroperitoneum". Considering that the tumor is locally retroperiton ". Con il 18, 2015, May 11, 2015, Jue 2, 2015, June 29, 2015 and August 2, 2015, neoadjuvant chemotherapy was given to "anzatax 210 mgivgttd $1+$ oxaliplatin (aiheng) $150 \mathrm{mg}$ ivgttd2 + Xeloda $1000 \mathrm{mg}$ bidpod1-14", supplemented by antiemetic, acid making, stomach protection, liver protection and nutritional support.)

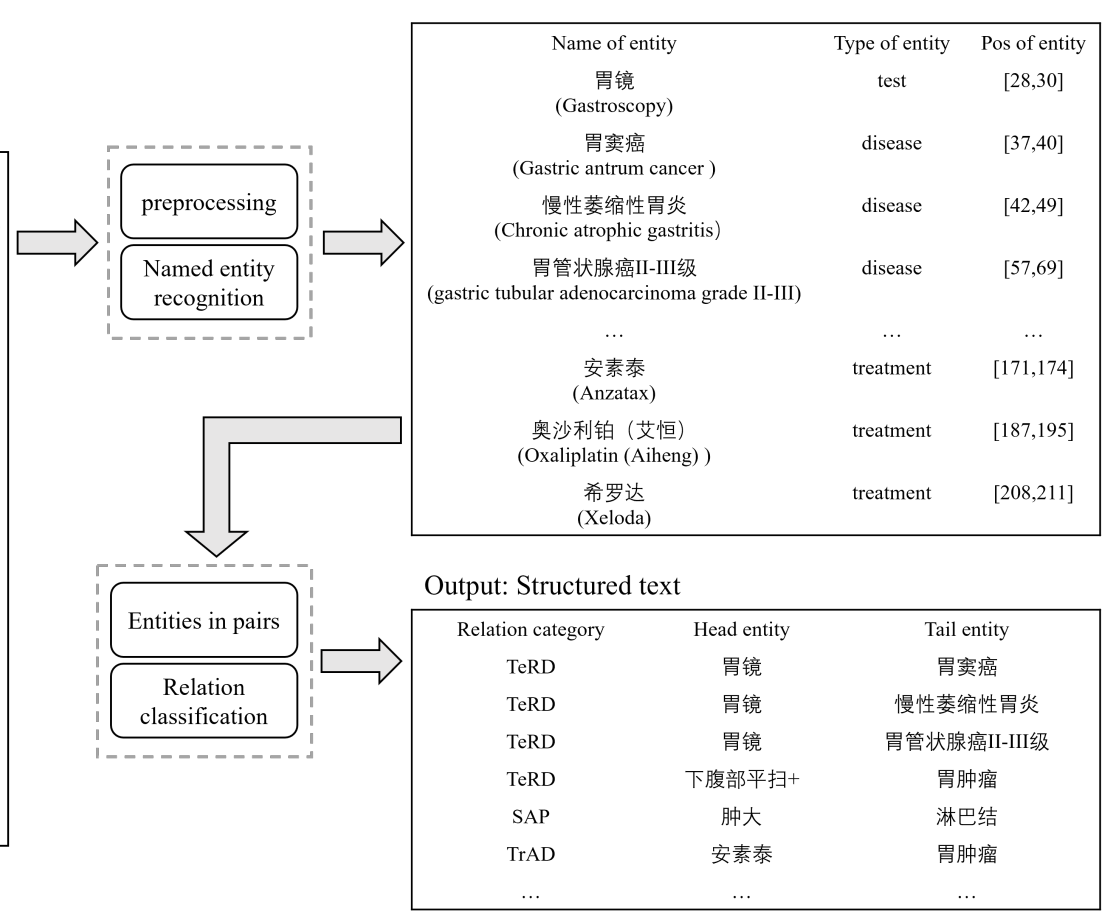

ResGRU: This model is similar to our model, except that it does not use the attention mechanism.

The attention mechanism used in our model is the same as in CNN-Att, BiLSTM-Att, and BiGRU-Att. We use Precision, Recall, and F1-score as the evaluation metrics of the experimental results. Experimental parameters used in the relation extraction model are shown in Table 3.

Table 3 Experimental parameters setting

\begin{tabular}{cc}
\hline parameters & value \\
\hline batch size & 64 \\
dimension of character embedding & 300 \\
dimension of position embedding & 25 \\
GRU hidden units & 512 \\
GRU hidden layer & 3 \\
window size & $3,5,7$ \\
number of filters & 128 \\
learning rate & 0.015 \\
optimizer & Adam \\
dropout & 0.5
\end{tabular}

\section{Results}

Comparison of ResNet and CNN with different depths

To explore the influence of network depths on the performance of relation extraction model, we compared CNN with single-layer, 5-layer and ResNet with 5layer, 9-layer, 11-layer and 13-layer. This experiment was conducted in the training set and test set with a total data of 17000 and evaluated by calculating the overall F1 value of all relationships.

Figure 7 shows that CNN-5 is inferior to the baseline $\mathrm{CNN}$, which indicates that directly increasing the depths of network layers on CNN is prone to overfitting and performance degradation. The results of ResNet-9, ResNet-11, and ResNet-13 are significantly better than that of CNN and CNN-5. This is because ResNet utilizes the shortcut connections between network layers to better integrate shallow and deep features, and improve the generalization ability of the model. The results of ResNet-5 and CNN-5 are close, 
Figure 7 Example of relation extraction

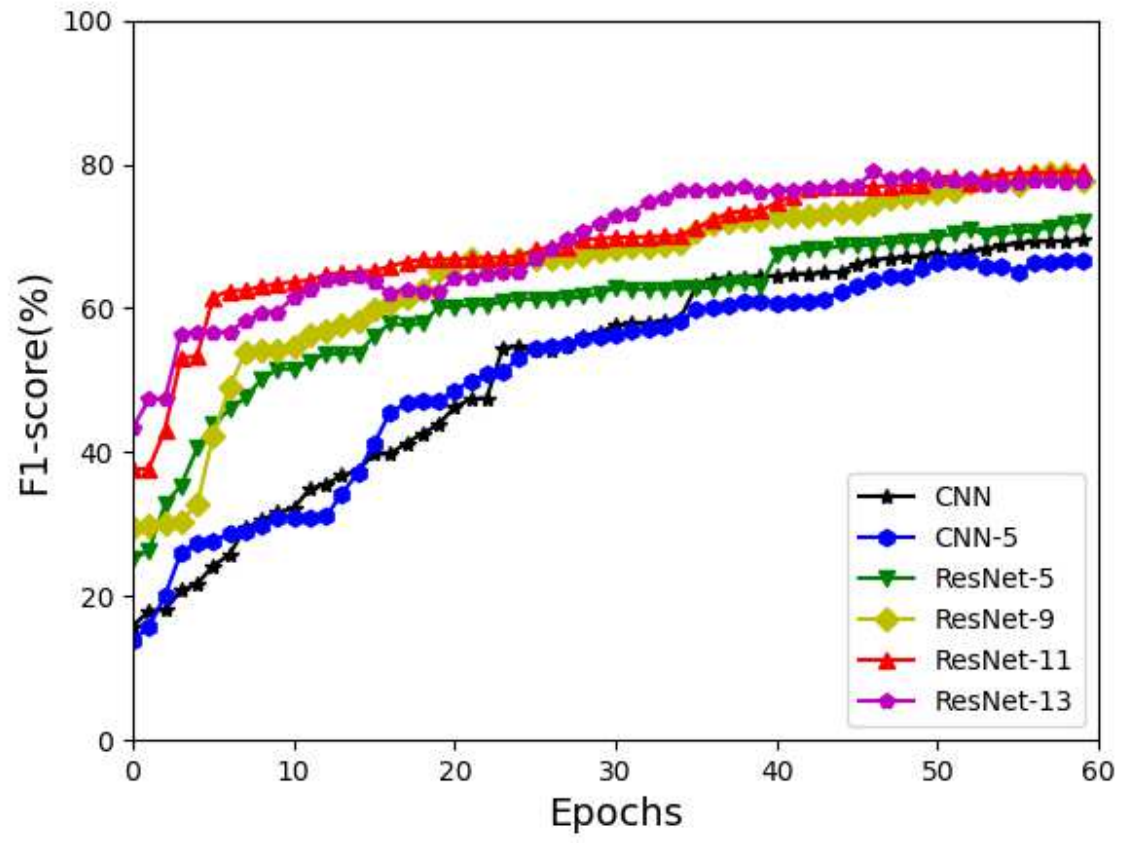

which shows that ResNet has little effect on shallow networks. As the depth of network increases, the performance of ResNet gradually increases, and the performance reaches saturation when the number of network layers is up to 11 .

In the subsequent experiment, we use the 11-layer ResNet to reduce the amount of calculation and memory usage as much as possible, while ensuring that the model can achieve better performance.

\section{Bootstrapping experiment}

To verify the performance of bootstrapping algorithm, we verify the overall $\mathrm{F} 1$ score of multiple models under the increasing amount of data.

Table 4 shows the F1-score of all models on different scale datasets. It can be seen that as the datasets increases, the F-score of all models has been significantly improved. In the two training stages of the datasets increasing from $\mathrm{N}=17000$ to $\mathrm{N}=30000$ and from $\mathrm{N}=30000$ to $\mathrm{N}=45000$, the model performance improved the most. After the datasets increased to 60000 , the model performance gradually became saturated, and the datasets stop training when it reaches 75000. Compared with the experimental results on the initial datasets, the F1-score of the CNN-Att increased by $10.57 \%$, BiLSTM-Att increased by $9.09 \%$, and the ResGRU-Att proposed in this paper increased by $7.52 \%$. Except for the PCNN, the F1-score of all models has increased by more than $7 \%$.

The ResGRU-Att has achieved the best results on both the initial and final datasets, and the F1-score has always remained above $80 \%$. This shows that bootstrapping algorithm is suitable for expanding data. However, as the amount of data increase, bootstrapping still inevitably has the problem of semantic drift due to some ambiguous annotations, which is also a major disadvantage of bootstrapping. In terms of relationship extraction, the learning effect of our model is better.

Why PCNN performance does not improve much when the amount of data increases? PCNN is an improved CNN to solve the problems of labeling errors in relation extraction using remote supervision and noise in feature extraction [11]. PCNN is much better than CNN when the amount of data is only 17000 . Because PCNN is trying to avoid this problem, but the increasing data hinders the model learning, the model effect of PCNN is the worst.

Since the model achieves the best effect when the amount of data is 75000 , to ensure better performance of the model and reduce the amount of calculation and memory use, we will verify it when the amount of data is 75000 in subsequent experiments. 
Table 4 Comparison of F1-score of all models on different scale datasets

\begin{tabular}{|c|c|c|c|c|c|}
\hline Models & $\mathrm{N}=17000$ & $\mathrm{~N}=30000$ & $\mathrm{~N}=45000$ & $\mathrm{~N}=60000$ & $\mathrm{~N}=75000$ \\
\hline CNN & 67.99 & 69.67 & 72.8 & 74.86 & 76.71 \\
\hline CNN-Att & 70.49 & 73.57 & 75 & 78.44 & 81.06 \\
\hline PCNN & 74.46 & 75.24 & 77.8 & 78.26 & 79.57 \\
\hline ResNet & 78.27 & 79.75 & 81.52 & 83.34 & 86.13 \\
\hline BiLSTM-Att & 76.12 & 80.98 & 82.71 & 84.96 & 85.21 \\
\hline BiGRU-Att & 77.96 & 81.18 & 83.9 & 85.11 & 85.94 \\
\hline ResGRU & 80 & 84.08 & 86 & 86.74 & 87.09 \\
\hline ResGRU-Att & 82.26 & 85.1 & 88.48 & 89.27 & 89.78 \\
\hline
\end{tabular}

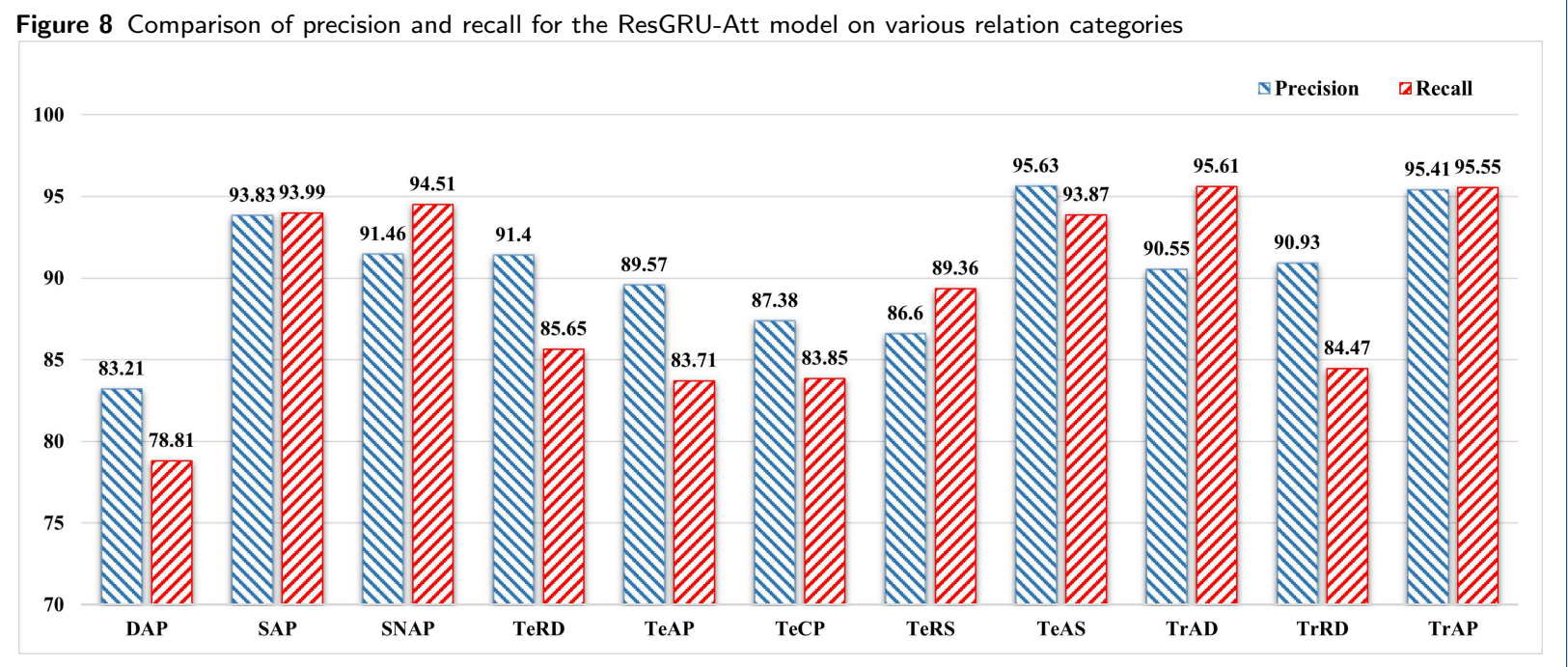

\section{Comparison with Prior Work}

Figure 8 shows the accuracy and recall of the ResGRUAtt on specific relation categories. It can be found that ResGRU-Att achieves the highest accuracy rate on the category TrAS, reaching 95.63\%, and the highest accuracy rate on the category TrAP, reaching $95.55 \%$. Combining the two evaluation metrics of precision and recall, the ResGRU-Att performs best in the three relation categories between SAP, TeAS, and TrAP, with recall and precision exceeding $93 \%$, and poor performance on DAP and TrRD, the precision and recall do not reach $85 \%$.

Why does the same model perform differently in different relationship categories? We find that the symptom entities and test entities are densely distributed in the data set, and the composition structure is relatively single. The number of disease and treatment entities is small, the structure is complex, the boundary is fuzzy and the entities are nested. For example, the entity "消化道出血原因待查 2 、重度贫血 3 、 肝硬化 4 、慢性丙型肝炎[the cause of gastrointestinal bleeding remains to be investigated 2 , severe anemia 3 , liver cirrhosis 4, chronic hepatitis C]" is wrongly identified as "gastrointestinal bleeding", "severe anemia" and other entities. On the other hand, position-related entities often appear in the interior of treatment and disease. These three types of entities are prone to entity type ambiguity in the process of recognition. For example, the "消化道[digestive tract]" in the example above is actually a position entity, but the whole large entity is actually a disease.

When extracting relations, the above situation affects the effect of relation extraction and also makes the same model appear dissimilar in different relations. This difference is related to the number, length and composition of the entities themselves.

Table 5 Comparison of performance for different models on overall relation categories

\begin{tabular}{cccc}
\hline Models & Precision & Recall & F1-score \\
\hline CNN & 79.39 & 74.21 & 76.71 \\
CNN-Att & 85.46 & 77.09 & 81.06 \\
PCNN & 83.56 & 75.94 & 79.57 \\
ResNet & 88.44 & 83.94 & 86.13 \\
BiLSTM-Att & 85.4 & 85.02 & 85.21 \\
BiGRU-Att & 87.75 & 84.20 & 85.94 \\
ResGRU & 86.47 & 87.72 & 87.09 \\
ResGRU-Att & $\mathbf{9 0 . 5 4}$ & $\mathbf{8 9 . 0 3}$ & $\mathbf{8 9 . 7 8}$ \\
\hline
\end{tabular}

Table 5 shows the comparison of different models on the overall relation categories. We can see that the ResGRU-Att has achieved the best experimental results, with the accuracy, recall and F1-score reaching $90.54 \%, 89.03 \%$ and $89.78 \%$ respectively. Compared 
with the baseline model, the F1-score increased by $13.07 \%$. Compared with the BiGRU-Att, it has increased by $3.84 \%$. and F1 value increases by $3.65 \%$ compared with ResNet, which shows that the hybrid neural network combining ResNet and BiGRU has a better effect on feature extraction than ResNet and BiGRU alone. The ResGRU and the ResNet also perform well without using the attention mechanism, with the F1-score reaching $87.09 \%$ and $86.13 \%$ respectively. which shows that the residual block in the residual network has a good ability to transmit information. The ResGRU that uses the hybrid neural network as the feature extractor performs better than the ResNet, and the F1-score is $0.96 \%$ higher. It shows that the hybrid structure of the gated cyclic network has stronger information retention ability. ResGRU-Att is $2.69 \%$ higher than ResGRU's F1 value, indicating that the judgment of relationship types by different characters is very important, and the attention mechanism can further highlight the critical information of relationship classification, so our model also achieves the best effect.

Table 6 shows the comparison of F1-score of different models on specific relation categories. Due to previous experiments, we know that the number and distribution of entities are different, and some entities still have problems such as fuzzy boundary and nesting. The number of relationship categories, the distance between entity pairs and the complexity of sentence semantics lead to dissimilar effects of the model on different relationship categories.

Combined with the overall results, most models perform best on SAP and TrAD, but poorly on DAP. The ResGRU-Att has achieved better results than other models in nine categories of relation, and the F1-score on all relation exceed $80 \%$, with the F1score on SAP, SNAP, TeAS, TrAD and TrAP reaching $93.91 \%, 92.96 \%, 94.74 \%, 93.01 \%$ and $95.48 \%$ respectively. The best results on the other two relation categories of TeAP and SNAP are obtained by the BiGRU and the ResGRU, with the F1-score reaching $89.93 \%$ and $93.37 \%$. Compared with the baseline CNN, the ResGRU-Att has the greatest improvement on the four relation categories of DAP, SAP, SNAP, and TeAS, with F1-score increased by $16.09 \%, 15.87 \%, 17.01 \%$ and $7.56 \%$.

\section{Discussion}

Based on the experimental results of the above different models, the following conclusions can be drawn:

(1) The ResNet, ResGRU and ResGRU-Att of the residual network are used to obtain better results than a single CNN and RNN. The reason is that the deep ResNet has a stronger feature extraction ability than the shallow network, which can avoid the overfitting problem of the baseline CNN.

(2) The bootstrapping semi-supervised algorithm is suitable for expanding the Chinese EMRs relation corpus, and the recognition accuracy on various relation categories has been significantly improved.

(3) The ResGRU-Att with attention mechanism is better than the ResGRU, which shows that the judgment of relationship types by different characters is very important, and the attention mechanism can improve the performance of model.

(4) The ResGRU-Att model proposed in this paper combines the advantages of ResNet and GRU neural networks and performs well in the overall relation categories.

The ResGRU-Att model shows differences in the extraction results of different relation categories. The reason may be related to the number of different relation categories, the distance between entity pairs, and the complexity of sentence semantics. Secondly, while using the bootstrapping algorithm to expand the training set, some relation categories introduce more noise, which will cause certain interference to relation extraction.

\section{Conclusions}

In this study, we introduce a hybrid neural network method based on semi-supervised learning to extract entity relations from Chinese EMRs. This method firstly uses a residual network to reduce information loss during feature transmission and combines bidirectional GRU to capture long-term dependency and attention mechanisms to highlight key information. We train with a small amount of relation datasets annotated manually and use the bootstrapping algorithm to continuously expand the datasets. F1-score of our model exceeds $90 \%$ on five of the pre-defined relation categories and reaches $89.78 \%$ on the overall relations. Experimental results show that our method is suitable for extracting the relations between medical entities in Chinese EMRs. In the future study, we will attempt to add additional features and use the joint model or pre-trained language model to further improve the performance of relation extraction model.

\section{Declarations}

Acknowledgments

We would like to thank CCKS for providing the original case corpus in the study.

\section{Funding}

This study is supported by the Sichuan Science and Technology Program (NO. 2020YFS0057,2021YFG0031)

\section{Authors' contributions}

Chunming $Y$ and Dan $X$ participated in all aspects of the study, including study design, data collection and analysis, and drafting the manuscript. All authors analyzed the data and were involved in writing the manuscript. 
Table 6 Comparison of F1-score for different models on various relation categories

\begin{tabular}{cccccccccccc}
\hline Models & DAP & SAP & SNAP & TeRD & TeAP & TeCP & TeRS & TeAS & TrAD & TrRD & TrAP \\
\hline CNN & 64.86 & 78.04 & 75.95 & 79.51 & 85 & 74.29 & 80.4 & 66.76 & 83.9 & $\mathbf{7 2 . 7 4}$ & 82.36 \\
CNN-Att & 70.33 & 82.89 & 84.79 & 77.16 & 84.98 & 80.1 & 84.89 & 75.67 & 83.73 & 83.71 & 83.42 \\
PCNN & 69.53 & 84.74 & 80.06 & 75.69 & 82.28 & 76.36 & 79.95 & 75.43 & 84.74 & 83.44 & 83.05 \\
ResNet & 75.98 & 91.11 & 86.95 & 84.18 & 89.89 & 78.59 & 85.86 & 86 & $\mathbf{9 4 . 9 9}$ & $\mathbf{8 7 . 6 2}$ & 86.24 \\
BiLSTM-Att & 74.96 & 92.83 & 92.48 & 84.84 & 85.27 & 73.57 & 85.6 & 87.47 & 92.48 & 80.34 & 87.43 \\
BiGRU-Att & 75.34 & 90.62 & 90 & 87.43 & $\mathbf{8 9 . 9 3}$ & 83.18 & 85.27 & 81.84 & 92.08 & 82.24 & 87.44 \\
ResGRU & 78.53 & $\mathbf{9 2}$ & $\mathbf{9 3 . 3 7}$ & $\mathbf{9 2 . 0 5}$ & 85 & 83.39 & 84.46 & 80.13 & 91.83 & 87.11 & 90.17 \\
ResGRU-Att & $\mathbf{8 0 . 9 5}$ & $\mathbf{9 3 . 9 1}$ & $\mathbf{9 2 . 9 6}$ & $\mathbf{8 8 . 4 3}$ & $\mathbf{8 6 . 5 4}$ & $\mathbf{8 5 . 5 8}$ & $\mathbf{8 7 . 9 6}$ & $\mathbf{9 4 . 7 4}$ & 93.01 & 87.58 & $\mathbf{9 5 . 4 8}$ \\
\hline
\end{tabular}

\section{Availability of data and materials}

The data and code that support the findings of this study are available from https://github.com/yangchunm/Relation-Extaction

Ethics approval and consent to participate

Not applicable

\section{Competing interests}

No competing interests exists at the time of submission of this manuscript.

\section{Consent for publication}

Not applicable

\section{Author details}

${ }^{1}$ School of Computer Science and Technology, Southwest University of Science and Technology, Mianyang 621010, Sichuan, China. ${ }^{2}$ School of Science, Southwest University of Science and Technology, Mianyang 621010, Sichuan, China. ${ }^{3}$ Sichuan Big Data and Intelligent Systems Engineering Technology Research Center, Mianyang 621010, Sichuan, China.

\section{References}

1. Yang, J., Guan, Y., He, B., Qu, C., Yu, Q., Liu, Y., Zhao, Y.: Corpus construction for named entities and entity relations on chinese electronic medical records. J Softw 27(11), 2725-46 (2016)

2. Zhang, Z., Zhou, T., Zhang, Y., Pang, Y.: Attention-based deep residual learning network for entity relation extraction in chinese emrs. BMC medical informatics and decision making 19(2), 171-177 (2019)

3. Huang, M., Li, M., Han, H.: Research on entity recognition and knowledge graph construction based on electronic medical records. J. Application Research of Computers 36(12) (2019)

4. Liu, L., Li, B., Zhang, X.: Named entity relation extraction based on svm training by positive and negative cases. J Comput Appl 28(6), 1444-1437 (2008)

5. Sam, R.C., Le, H.T., Nguyen, T.T., et al.: Relation extraction in vietnamese text using conditional random fields. In: Asia Information Retrieval Symposium, pp. 330-339 (2010). Springer

6. Zhang, H., Hou, S., Xia, X.: A novel convolution kernel model for chinese relation extraction based on semantic feature and instances partition. In: 2012 Fifth International Symposium on Computational Intelligence and Design, vol. 1, pp. 411-414 (2012). IEEE

7. Liu, C., Sun, W., Chao, W., Che, W.: Convolution neural network for relation extraction. In: International Conference on Advanced Data Mining and Applications, pp. 231-242 (2013). Springer

8. Nguyen, T.H., Grishman, R.: Relation extraction: Perspective from convolutional neural networks. In: Proceedings of the 1st Workshop on Vector Space Modeling for Natural Language Processing, pp. 39-48 (2015)

9. Ebrahimi, J., Dou, D.: Chain based rnn for relation classification. In Proceedings of the 2015 Conference of the North American Chapter of the Association for Computational Linguistics: Human Language Technologies, pp. 1244-1249 (2015)

10. Miwa, M., Bansal, M.: End-to-end relation extraction using Istms on sequences and tree structures. arXiv preprint arXiv:1601.00770 (2016)

11. Zeng, D., Liu, K., Chen, Y., Zhao, J.: Distant supervision for relation extraction via piecewise convolutional neural networks. In: Proceedings of the 2015 Conference on Empirical Methods in Natural Language Processing, pp. 1753-1762 (2015)

12. Lin, Y., Shen, S., Liu, Z., Luan, H., Sun, M.: Neural relation extraction with selective attention over instances. In: Proceedings of the 54th
Annual Meeting of the Association for Computational Linguistics (Volume 1: Long Papers), pp. 2124-2133 (2016)

13. Zhou, P., Shi, W., Tian, J., Qi, Z., Li, B., Hao, H., Xu, B. Attention-based bidirectional long short-term memory networks for relation classification. In: Proceedings of the 54th Annual Meeting of the Association for Computational Linguistics (volume 2: Short Papers), pp. 207-212 (2016)

14. He, K., Zhang, X., Ren, S., Sun, J.: Deep residual learning for image recognition. In: Proceedings of the IEEE Conference on Computer Vision and Pattern Recognition, pp. 770-778 (2016)

15. Hong, L., Lin, J., Li, S., Wan, F., Yang, H., Jiang, T., Zhao, D., Zeng, J.: A novel machine learning framework for automated biomedical relation extraction from large-scale literature repositories. Nature Machine Intelligence 2(6), 347-355 (2020)

16. Uzuner, Ö., South, B.R., Shen, S., DuVall, S.L.: 2010 i2b2/va challenge on concepts, assertions, and relations in clinical text. Journal of the American Medical Informatics Association 18(5), 552-556 (2011)

17. Christopoulou, F., Tran, T.T., Sahu, S.K., Miwa, M., Ananiadou, S.: Adverse drug events and medication relation extraction in electronic health records with ensemble deep learning methods. Journal of the American Medical Informatics Association 27(1), 39-46 (2020)

18. Xu, D., Zhang, M., Zhao, T., Ge, C., Gao, W., Wei, J., Zhu, K.Q.: Data-driven information extraction from chinese electronic medical records. PloS one 10(8), 0136270 (2015)

19. Zhang, Y., Lu, Z.: Exploring semi-supervised variational autoencoders for biomedical relation extraction. Methods 166, 112-119 (2019)

20. Song, L., Zhang, Y., Gildea, D., Yu, M., Wang, Z., Su, J.: Leveraging dependency forest for neural medical relation extraction. arXiv preprint arXiv:1911.04123 (2019)

21. LIU Ningning, X.X.W.J.Z.R. JU Shenggen: Drug-drug relationship extraction based on capsule networks. Journal ofChineselnformation Processing 34(1), 80-86 (2020)

22. Zeng, D., Liu, K., Lai, S., Zhou, G., Zhao, J.: Relation classification via convolutional deep neural network. In: Proceedings of COLING 2014, the 25th International Conference on Computational Linguistics: Technical Papers, pp. 2335-2344 (2014)

23. Huang, Y.Y., Wang, W.Y.: Deep residual learning for weakly-supervised relation extraction. arXiv preprint arXiv:1707.08866 (2017)

24. China Conference on Knowledge Graph and Semantic Computing (2017). http://www.sigkg.cn/ccks2017/ ?page $_{i} d=$ 51 AccessedAccessed 20 May 2017

25. China Conference on Knowledge Graph and Semantic Computing (2019). http://www.sigkg.cn/ccks2019/? page $_{i} d=$ 62 AccessedAccessed20April2019

26. China Conference on Knowledge Graph and Semantic Computing (2020). http://sigkg.cn/ccks2020/ ?page $_{i} d=$ 516 AccessedAccessed 20 May 2020 